\title{
Hearing assessment in autistic spectrum disorder; is it unnecessary and pointless procedure in developing countries?
}

\author{
Adel M. Zeglam ${ }^{1,2 *}$ and Samah M. Al-Ksaik ${ }^{1,2}$ \\ ${ }^{1}$ Consultant Neurodevelopment Pediatrician, Professor of Pediatrics and Child Health, Al-Khadra Teaching Hospital, Tripoli University, Tripoli, Libya \\ ${ }^{2}$ Pediatric SHO, Al-Khadra Hospital, Tripoli, Libya
}

\begin{abstract}
Aims: to examine current medical practice of ordering ABR for all children with ASD, assess the evidence of the value of this test in these children and its applicability in developing countries to which Libya belongs.

Method: The medical records of all children with ASD presented to neurodevelopment clinics over five year's period (January 2010 to December 2014 ) were reviewed.

Results: in 40 children out of 2006 children with ASD, the families were concerned about their children hearing and reaction to load noises. Auditory Brain Stem Response (ABR) confirmed that 23 out of these 40 children had Sensory Neural Hearing Loss (SNHL).

Conclusion: In developing countries where the delivery of effective public health interventions to people in need is compromised, offering a "less is more" attitude might be very effective. Infants and children with ASD are assessed according to local protocols. ABR is considered unnecessary in those without clinical signs suggesting hearing impairment and without any parental concerns.
\end{abstract}

\section{Introduction}

In developing and poor countries where the health care is in a state of anarchy; spending money on unaffordable and probably unnecessary investigation might be substituted by a reliable, simple and more informative tool that can deal with the problem.

Mental illness is the leading cause of disability in the world, according to the World Health Organization. Even more troubling: Four out of five people with psychiatric or neurodevelopmental disorders live in developing countries, where they have few opportunities for treatment.

As stated in the report of the Commission on Social Determinants of Health [1]: "Poverty is not only lack of income. The implication, both of the social gradient in health and the poor health of the poorest of the poor, is that health inequity is caused by the unequal distribution of income, goods, and services and of the consequent chance of leading a flourishing life. This is not in any sense a 'natural' phenomenon."

A substantial part of the medicine we practice is preserved in code of practices. The assessment and management of children with Autistic Spectrum Disorder (ASD) is no exemption. At regular intervals, the need and requirement for new recommendations has been re-examined in the medical press. On the other hand, despite the prevalence of this clinical problem, agreement over management remains contested. In particular, the need for hearing test in children with ASD continues. According to the fifth edition of Diagnostic and Statistical Manual of the American Psychiatric Association [2]; (ASD) is a lifelong developmental disability defined by diagnostic criteria that include deficits in social communication and social interaction and restricted, repetitive patterns of behavior, interests, or activities.
New USA government survey (2014) of parents suggests that 1 in 45 children, aged 3 through 17, had been diagnosed with ASD. This is notably higher than the official government estimate of 1 in 68 American children with autism, as stated by National Center for Health Statistics [3].

In Libya, One prospective hospital-based study [4] carried out on all children referred to a neurodevelopment clinic between 2005 and 2009 for ASD assessment showed a prevalence of one in 300. $(3,4)$. In the 2011 study 200 children whose ages "ranged from younger than 3 years to over 12 years" were referred for ASD assessment. ASD were diagnosed in $83 \%$ of cases (166 children) which gives a prevalence of 10:1000. The prevalence of the problem is probably higher or similar to that seen in the USA and UK. No data were available from the Arab countries or other developing countries [5].

The rising estimate of the number of children with autism does not include children who are deaf. According to the Annual Survey of Deaf and Hard of Hearing Children and Youth, which is conducted by the Gallaudet Research Institute in the survey for [7]; Results indicate that 1 in 59 children (specifically 8-year olds) with hearing loss were also receiving services for autism [8]; considerably higher, than reported

Correspondence to: Adel M. Zeglam, Consultant Neurodevelopment Pediatrician, Professor of Pediatrics and Child Health, Al-Khadra Teaching Hospital, Tripoli University, Tripoli, Libya, Tel: (267) 426-8337; E-mail: adelzeglam@gmail.com

Received: August 02, 2016; Accepted: August 19, 2016; Published: August 23 2016 
national estimates of 1 in 91 [9] and 1 in 110 [10] for hearing children. This is approximately twice what is currently believed to be the national prevalence rate, 1 in 150 [11].

On average, a child who is deaf will be diagnosed with autism later in life than a hearing child with autism. In one study, deaf children were diagnosed an average of 1 year later: [12]. Researchers and educators speculate that the reason behind delayed diagnoses is the difficulty encountered in distinguishing characteristics of deafness from characteristics of autism, as well as limited resources for parents and educators guiding the identification of autism and deafness. Also, it is possible that deaf children are diagnosed later because there are relatively few psychological tests that have been made for, or even include, considerations for children who are deaf. In fact, there are no approved instruments for making a diagnosis of autism in a child who is deaf [13]. Some suggest that children with autism have language difficulties because they struggle with cross-modality-converting auditory information to vocal utterances [14]. Others have suggested that children with autism have severe auditory processing deficits [15].

Deaf child might be occupied by self-stimulating behavior and plays independently, this does not mean the child is autistic but simply he/she cannot hear other children. Knowing if the child has hearing impairment is not always apparent. The fact that hearing loss often is not diagnosed until a child is 2 or 3 years old-the same time that autism is often diagnosed in children-increases the difficulty of assessment. Distinctive features of autism may mimic deafness and vice versa. If a child cannot hear, he or she will not respond when called by name-often an important item on checklist of autism, but also typical of deafness. Additionally if a child cannot hear, social interaction with peers might be difficult-due to an inability to hear conversation, not because he or she has autism. Healthcare in developing countries in general differs widely, depending on the country and also the region those living in urban areas are more likely to receive better healthcare services than those in rural or remote regions. Hospitals and clinics in Africa often find it difficult to employ enough trained medical staff to cope with the number of people needing care. Infrastructure problems have made it difficult to provide services to many people in more remote areas [16].

Lack of access by people living in low income countries may deprive them of early detection and reduces opportunities of early intervention. Diagnostic laboratories are often poorly resourced in developing countries, and sparsely distributed. Many developing countries with weak and poorly resourced health care systems are burdened with the poor levels of primary healthcare systems detrimental to their socioeconomic development.

Laboratories in developing countries are often sparsely distributed, and access may be limited by economic or geographical factors. Where they do exist clinical laboratories are often under resourced and amenities such as electrical supply and water may be unreliable [17]. Shortage of skilled technical personnel is also a problem in some countries, particularly in rural areas. Particular diagnostic tests may not be available to the majority population due to their high cost or lack of robustness. In addition, some manufacturers may be reluctant to supply countries if return on their investment is likely to be low or where it may be difficult to establish effective mechanisms for product distribution or technical support [18].

Healthcare systems across developing countries including Libya face a number of formidable challenges. Limited energy resources, inaccessibility to medical facilities, limited government funding, and high costs are placing tremendous strain on the healthcare system in general and on children with special needs in particular.

In view of all these difficulties, this study examines current practice, and the evidence of the value of hearing tests in children with ASD in Tripoli Libya.

\section{Methodology}

\section{Design}

This is a hospital-based study where a range of assessment tools were used to diagnose ASD, including the Modified Checklist for Autism in Toddlers (M-CHAT) and Reviewed Autism Diagnostic Interview (ADI-R) and observations in various settings and discussions with other professionals. Diagnoses was based on the assessment results and according to DSM-IV \& V criteria.

Two types of tests that were used in screening for hearing loss. Auditory Brainstem Response (ABR) and Otoacoustic Emission Test (OAE). All ABRs were acquired with a GSI 37 AUDIO screener which is a revolutionary hearing screening device, combining the two-in-one technology of otoacoustic emissions (OAE) and auditory brainstem response (ABR). For infants, only one sound is presented, which is called the "click." If a healthy result is obtained then the child passes the ABR. OAE measures the reaction that is produced by the inner ear or cochlea. If an emission is produced for sounds that are essential to speech comprehension then the child has passed the test.

The Neurodevelopment Clinics are dedicated clinics of a tertiary care University Hospital labeled as referral and diagnostic clinics for autism and other neurodevelopmental problems. There are three regular weekly clinics. These clinics are consultant-led (AZ) in addition to having one registrar, two senior house officers and nurses. The clinics serve Tripoli, its suburbs and other district hospitals. The clinics receive referrals from other regions in Libya and care for children with other neurodevelopmental problems, hypotonia and epilepsy in the region. An average of 40 children are seen in each session. The age ranges from one month to sixteen years. About $70 \%$ of the children who attend the clinics are for Autism and speech and language disorders assessment.

The exclusion criteria were: 1) known neuro-Metabolic disorders, genetic or infectious disease, 2) chromosomal abnormalities, 3) diagnosed neurological or neurodegenerative disorders 4) major physical abnormalities and congenital malformation.

The inclusion criteria were pediatric age group (range: 1-16 years), all children included must have speech and language disorders and were diagnosed as ASD and/or SNHL.

All children underwent complete general, neurological and neurodevelopment assessment; the results of the general and neurological examination were unremarkable but the results of the neurodevelopment assessment were variable and needed to be reevaluated in a separate session.

For each child, the parent was asked to respond to a brief demographic and social questionnaire covering the child's age, gender, birth order, number of siblings, whether there were one or two parents at home, and whether the child had received professional psychological help.

When evaluating listening skills, observations are key. The family were asked if a child does not respond to an auditory stimulus, such as his/her name by turning their head to maintain eye contact upon 
hearing it. Do they respond in other ways? Do they change their facial expression or make an eye shift when they hear their name? Do they turn away? Do they respond verbally?

Data was collected over a five year's period (from January 2010 to December 2014). Due to high illiteracy rates among rural parents, questionnaires were administered through interviews at the clinic. The interviewers were either the registrar or any of the junior doctors attending the clinic who were given instructions by the investigator (AZ) about how to ensure consistency and avoid bias in filling the questionnaires.

Due to the high cost of the ABR, the control ABR's results 700 (440 male and 260 females) were retrospectively selected from records of children referred to the audiology department with speech and language disorders only (stammering, trauma, adenoids,) and without any neurological and neurodevelopmental disorders based upon the examination results that the child had before referral to the audiology clinic.

The following criteria to choose the control group were used:

1) Age matching (pediatric) with ASD children group, 2) ABR results including all stages performed in ASD children and 3) clinical files providing details that the neurological and neurodevelopmental examination were unremarkable and had been documented and confirmed by a consultant.

All information obtained was entered on specially designed forms; and kept in the medical files department.

An Ethics panel approval for the study was obtained, with all examinations of autistic children were performed in the presence of their parents. No consent is required for the control group as only patient files were used.

All statistical analyses were conducted using the SPSS statistical package.

\section{Results}

Total number of children seen between 2010 and 2014 were 306748. 2006 children were diagnosed with ASD giving a prevalence of 7:1000. Total number of children with SNHL were 75 out of whom 23 children had ASD. Prevalence of SNHL among Children with ASD was 11:1000 (1.1\%) while the prevalence of SNHL among all children seen in NDC $=25: 10000$. There are a limited number of studies that are looking at the incidence rate of SNHL and ASD, but the range in the existing literature of a dual diagnosis was exhibited by approximately $1 \%$ to $6 \%$ of children who are deaf or hard of hearing.

Rosenhall and colleagues found the incidence of SNHL among children with ASD as follows: $1.6 \%$ unilateral, $7.9 \%$ mild to moderate and $3.5 \%$ profound [19]. Other studies found the incidence between 5.3 $\%$ [20] and $1.7 \%$ [21].

Looking at Table 1, two things are immediately obvious from the data. The first is the low incidence of reaction to load noises in children with SNHL (5\%). The second is the high rate of concern regarding hearing among parents of children with SNHL (94\%) with only $6 \%$ of parents had no concerns regarding child's hearing. Further examination of the data is possible. In this study $98 \%$ of the parents of children with ASD had no concerns regarding hearing supported by the fact that $99 \%$ of those children reacted to sounds and noises. Much of the existing literature comments on the socialization and pretend play. Autistic spectrum disorder in the absence of associated features (eye to eye contact, social interaction, pretend play skills, Preferences, changes in routine) is extremely uncommon. In children with developmental delay, almost all of them reacted to load noises and their families had no concerns regarding their hearing or behavior but were concerned about the delayed speech and language which was obviously part of the children's developmental delay.

Only $22 \%$ of children with ASD and SNHL reacted to load noises and at the same time $87 \%$ of parents had concerns regarding hearing. All of these children underwent ABR at the time of diagnosis. Most of them had other associated features of ASD.

Extrapolation of these data shows that in 40 children out of 2006 children with ASD, the family were concerned about their children's hearing and reaction to load noises. ABR confirmed that 23 out of these 40 children had SNHL. In a child with autistic spectrum disorder, with no signs and symptoms of hearing impairment and with no parental concerns, over $86 \mathrm{ABR}$ would have to be performed to detect one occult case of SNHL.

\section{Discussion}

Speech and Language delays and hearing disorders such as sensorial loss, hypersensitivity to sound and otitis media are a few examples of associations connected to autism [22].

The management of children with autistic spectrum disorder has changed dramatically over the past 15 years. However, there is still a wide variation in practice between clinics and individual neurodevelopment paediatricians in the management of this common yet complex disorder. More than $70 \%$ of children in whom ASD was suspected would have undergone unnecessary hearing assessment because of a perceived risk of occult SNHL in this group of children. Is this justified? The available data from our study would suggest that it is not. The risk of SNHL in the absence of other clinical signs and parental concerns is extremely small (less than one in one hundred). Furthermore, our study showed that relying on parent's observation and concerns are very sensitive (87\%) and specific (99\%) predictor of SNHL in this group of children, saving money on unnecessary investigation when resources are very limited.

The routine ABR in children with autism is probably unjustified and puts more burden on families; as well as the pain and distress caused to the child.

Our study has a number of weaknesses. We did not collect information from other audiology services in other regions, which meant we had no data on interventions provided at these centers. As none of these centers has ABR/ASSR equipment, we can only assume that children would be diagnosed by subjective means, which would usually take place at an older age. We feel that including data on these children would add weight to our study. We also did not collect data on the interval between diagnosis and the initiation of the interventions.

\section{Conclusion}

Returning to a point made at the beginning of this article, there is a need to develop an affordable and reliable tool for hearing assessment in resource constrained, medically underserved areas of developing nations.

There are many obstacles facing the supply of proper health service in developing countries. These obstacles include corruption in the supply of medical and therapeutic products, temperamental supply of unreasonably expensive costs, and wide fluctuation in quality and 
safety. Absence of social security nets to ensure that poor people get the services they require, poor financial management and lack of successful administration and management, all these have added to current circumstance where many children have difficulties in accessing and finding the essential care they need simply because they are expensive and unaffordable.

My suggested practice following the diagnosis of ASD in any child and where hearing impaired is suspected follows a "less is more" attitude, Infants are assessed according to local protocols and those without clinical signs suggesting hearing impairment and without any parental concerns are given open appointment. If no concerns has occurred and the child appears well, ABR is considered unnecessary. I think this might be applicable to all developing countries where the delivery of effective public health interventions to people in need is compromised and health clinics have an even greater struggle to offer services to local populations particularly in remote rural areas.

\section{Acknowledgement}

The authors extend great thanks and appreciation to all the parents for their support and to all those involved in running this study. Thanks to my doctor colleagues, senior and junior, for referring these children to the clinic. We gratefully acknowledge the contribution of Dr. F. Abouriana, Dr. S. Al-Hmadi and Dr. Marwa Al-Ogab for all their help. Special thanks to Dr.Salah Joumaa consultant audiologist who helped with analysis of the audiological results.

\section{"Compliance with ethical standards"}

In accordance with the ethical standards of the institutional and national research committee; an Ethics panel approval for the study was obtained, and all examinations of autistic children were performed in the presence of their parents. Informed consent was obtained from all individual participants included in the study. No consent is required for the control group as only patient files were used.

This study has not received any fund from any source.

Both authors declare that they have no conflict of interest.

\section{"What is already known?"}

- Autistic spectrum disorder and sensory-neural hearing loss can co-exist.

- Increasing prevalence of autistic spectrum disorder.

- Limited health resources in developing countries may hamper early detection and intervention of any disease.

\section{"What this study adds?"}

- There is a need to develop an affordable and reliable tool for hearing assessment in resource constrained, medically underserved areas of developing nations.

- "Less is more" attitude in developing countries should be a satisfactory and fair approach.

- Children with autism and without clinical signs suggesting hearing impairment and without any parental concerns are given open appointment. If no concerns has occurred and the child appears well, ABR is considered unnecessary.

\section{References}

1. Final report: closing the gap in a generation: health equity through action on the social determinants of health. Final report of the Commission on Social Determinants of Health. Geneva, World Health Organization, 2008.

2. American Psychiatric Association; 2013.American Psychiatric Association. Diagnostic and statistical manual of mental disorders. 5th ed. Arlington, VA.

3. CDC, National Center for Health Statistics. Estimated Prevalence of Autism and Other Developmental Disabilities Following Questionnaire Changes in the 2014 National Health Interview Survey, Number 87, November 1, 2015.

4. Zeglam AM, Maouna A (2012) Is there a need for a focused health care service for children with autistic spectrum disorders? A keyhole look at this problem in Tripoli, Libya. Autism 16: 337-339. [Crossref]

5. Zeglam AM, Maound AJ (2012) Prevalence of autistic spectrum disorders in Tripoli, Libya: the need for more research and planned services. East Mediterr Health $J$ 18: 184-188. [Crossref]

6. Zeglam Adel M.; Al-Bloushi Haneen (2001) Autism today in Libya: Is it a tip of an iceberg? (A comparative study). Middle East Journal of Family Medicine 10: 34-38.

7. Gallaudet Research Institute. (2007). Regional and national summary report of data from the 2009-2010 Annual Survey of Deaf and Hard of Hearing Children and Youth. Washington, DC: Gallaudet University.

8. Szymanski CA, Brice PJ, Lam KH, Hotto SA (2012) Deaf children with autism spectrum disorders. J Autism Dev Disord 42: 2027-2037. [Crossref]

9. MD Kogan, SJ Blumberg, LA Schieve, CA Boyle, JM Perrin, et al. (2009) Prevalence of Parent-Reported Diagnosis of Autism Spectrum Disorder Among Children in the US, 2007. Pediatrics 124: 1395-1403.

10. Centers for Disease Control and Prevention (2007b). CDC releases new data on autism spectrum disorders (ASDs) from multiple communities in the United States.

11. Zelazny K, Simms LJ (2015) Confirmatory factor analyses of DSM-5 posttraumatic stress disorder symptoms in psychiatric samples differing in Criterion A status. $J$ Anxiety Disord 34: 15-23. [Crossref]

12. Mandell DS, Novak MM, Zubritsky CD (2005) Factors associated with age of diagnosis among children with autism spectrum disorders. Pediatrics 116: 1480-1486. [Crossref]

13. Roper L, Arnold P, Monteiro B (2003) Co-occurrence of autism and deafness: diagnostic considerations. Autism 7: 245-253. [Crossref]

14. Bonvillian JD, Nelson KE, Rhyne JM (1981) Sign language and autism. J Autism Dev Disord 11: 125-137. [Crossref]

15. Ruttenberg BA, Wolf EG (1967) Evaluating the communication of the autistic child. $J$ Speech Hear Disord 32: 314-324. [Crossref]

16. 16. WHO: The World Health Report 2006 - Working together for health. Geneva 2006

17. Berkelman R, Cassell G, Specter S, Hamburg M, Klugman K (2006) The "Achilles heel" of global efforts to combat infectious diseases. Clin Infect Dis 42: 1503-1504. [Crossref]

18. 18. Peeling, R.; McNerney, R. Increasing Access to Diagnostics through Technology Transfer and Local Production; WHO: Geneva, Switzerland, 2011.

19. Rosenhall U, Nordin V, Sandström M, Ahlsén G, Gillberg C (1999) Autism and hearing loss. J Autism Dev Disord 29: 349-357. [Crossref]

20. Jure R, Rapin I, Tuchman RF (1991) Hearing-impaired autistic children. Dev Med Child Neurol 33: 1062-1072. [Crossref]

21. Levy SE, Giarelli E, Lee LC, et al. (2010) Autism spectrum disorder and co-occurring developmental, psychiatric, and medical conditions among children in multiple populations of the United States. J Dev Behav Pediatr 31: 267-275.

22. Davis R, Stiegler L (2011) Behavioral Hearing Assessment for Children with Autism (2010, July)

Copyright: (C2016 Zeglam AM. This is an open-access article distributed under the terms of the Creative Commons Attribution License, which permits unrestricted use, distribution, and reproduction in any medium, provided the original author and source are credited. 